\title{
SGDBR single-chip wavelength tunable lasers for swept source OCT
}

\author{
Dennis Derickson ${ }^{1}$, Mike Bernacil ${ }^{1}$, Andrew DeKelaita ${ }^{1}$, Ben Maher $^{1}$, Shane O'Connor ${ }^{1}$ \\ Matthew N. Sysak ${ }^{2}$ and Leif Johanssen ${ }^{2}$ \\ ${ }^{1}$ California Polytechnic State University, Electrical Engineering Department, \\ 1 Grand Avenue, San Luis Obispo, CA 93407 805-756-7584 ddericks@calpoly.edu \\ ${ }^{2}$ University of California at Santa Barbara, \\ Dept. of Electrical and Computer Engineering, Santa Barbara, CA 93106
}

\begin{abstract}
Sampled Grating Distributed Bragg Reflector (SGDBR) monolithic tunable lasers are now entering the production phase in telecommunications applications. These tunable lasers are unique in that they offer wide wavelength tuning $(1525$ to $1565 \mathrm{~nm})$, fast wavelength tuning $(5 \mathrm{~ns})$ and high speed amplitude modulation all on the same monolithic chip $^{1,2,3,4}$. This work studies the applicability of SGDBR monolithic tunable laser diodes for biomedical imaging using swept-wavelength or Fourier domain optical coherence tomography. This paper will present our work involved with utilizing the strengths (table 1) of this SGDBR laser class and mitigating the weaknesses (table 2) of this device for swept-wavelength imaging applications. The strengths of the laser are its small size (portable solutions), wide wavelength range (good distance resolution), fast switching speeds (improved update rates), wide choice of center wavelengths, and lower power consumption. The weaknesses being addressed are the complicated wavelength tuning mechanism (3 wavelength control currents), wider laser linewidth (10s of MHz), moderate output power (10mW ), and the need for improved laser packaging. This paper will highlight the source characterization results and discuss an initial measurement architecture utilizing the SGDBR measurement engine.
\end{abstract}

Keywords: semiconductor lasers, wavelength tunable lasers, optical coherence tomography, distributed Bragg reflector lasers,

Table 1: Strengths of the SGDBR laser for swept wavelength OCT

\begin{tabular}{|l|l|l|}
\hline & Strength Attribute & Implications of Strength \\
\hline 1 & Small size (3 mm chip length). & Portable instrumentation. \\
\hline 2 & 5 THz frequency tuning range. & Acceptable distance resolution. \\
\hline 3 & 5 ns frequency switching time. & Fast measurement update rates. \\
\hline 4 & Low cost. & More applications can be addressed cost effectively. \\
\hline 5 & Wavelength can be engineered. & 1550 and 1585 nm now, $800-1600 \mathrm{~nm}$ possible. \\
\hline 6 & Low power consumption. & Enhances portability and miniaturization. \\
\hline
\end{tabular}

Table 2: Weaknesses of the SGDBR laser for swept wavelength OCT

\begin{tabular}{|l|l|l|}
\hline Item & Weakness Attribute & Implications of Weakness \\
\hline 1 & $\begin{array}{l}\text { Complicated wavelength tuning mechanism (3 } \\
\text { input currents control the wavelength). }\end{array}$ & $\begin{array}{l}\text { High-speed driver circuit design effort is } \\
\text { required for a linear wavelength ramp. }\end{array}$ \\
\hline 2 & $\begin{array}{l}\text { Single continuous wavelength sweep not } \\
\text { available. }\end{array}$ & $\begin{array}{l}\text { Needs a system architecture that utilizes fast } \\
\text { stepped-tuning or ramp concatenations. }\end{array}$ \\
\hline 3 & Relatively wide laser line width $(10 \mathrm{MHz})$ & Must use architecture that tolerates weakness. \\
\hline 3 & Moderate output power (10 $\mathrm{mW})$. & May limit applications or require amplification. \\
\hline 4 & $\begin{array}{l}\text { Present commercial packaging may limit } \\
\text { wavelength tuning rate in some cases. }\end{array}$ & $\begin{array}{l}\text { Design the laser packaging for high speed } \\
\text { wavelength switching. }\end{array}$ \\
\hline
\end{tabular}




\section{INTRODUCTION OF THE SGDBR LASER}

Sampled Grating Distributed Bragg Reflector (SGDBR) single monolithic chip tunable lasers were developed for telecommunications applications ${ }^{1-4}$. These devices allow for wide wavelength tuning over the erbium-doped fiber amplifier operational band of 1525 to $1565 \mathrm{~nm}$ or the extended L-band of 1565 to $1605 \mathrm{~nm}$ Figure 1 shows a top-view photograph of an example SGDBR laser.

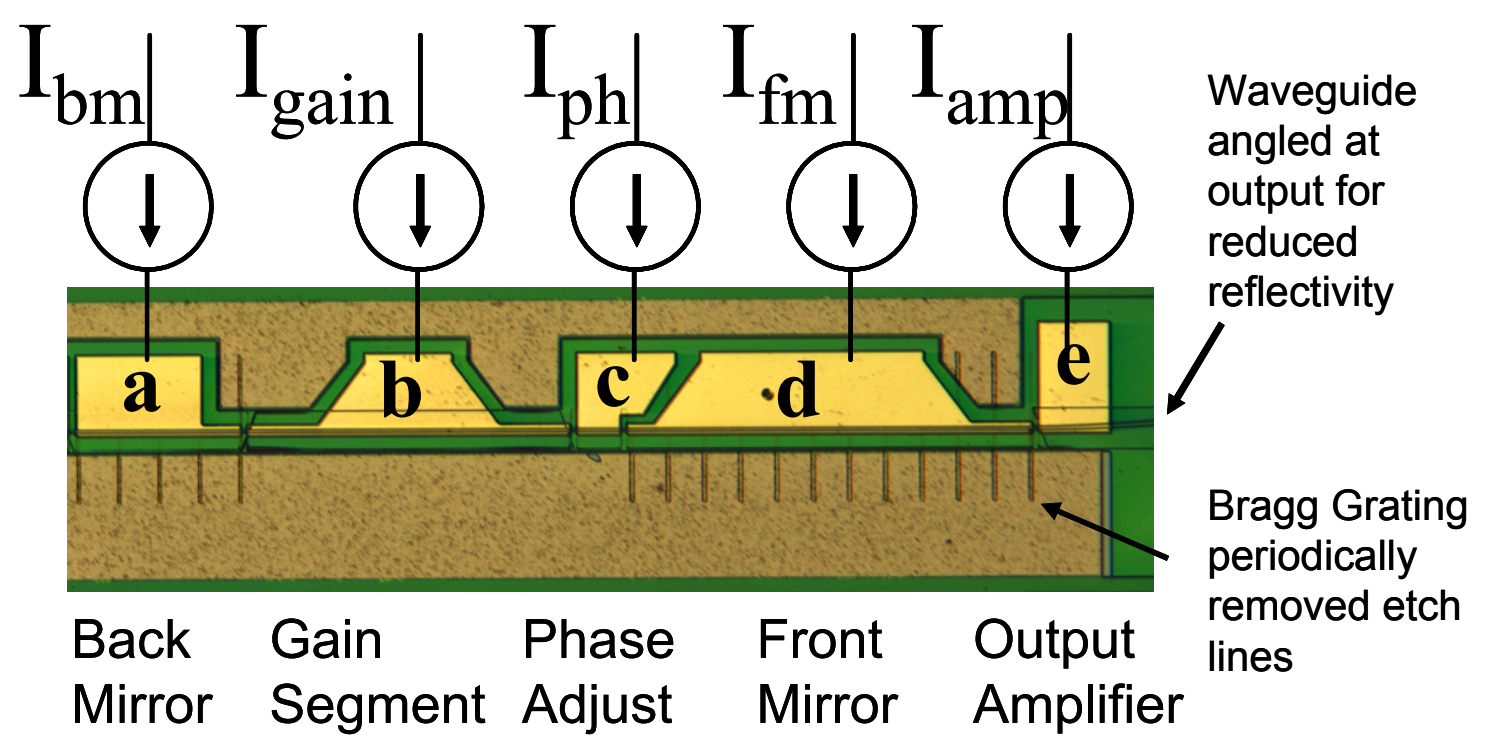

Figure 1: Photograph of a single chip tunable laser (top view). The bond pads from left to right are the (a) back mirror, (b) gain segment (c) phase shift segment, (d) front facet mirror and (e) output amplifier. The entire chip is about $3 \mathrm{~mm}$ long. Another variation of this device includes a monolithically integrated electroabsorption amplitude modulator.

The wavelength of the laser is controlled by three currents that are applied to the back mirror, front mirror, and phase sections of the laser. The cross sectional view of this laser is illustrated in Figure 2.

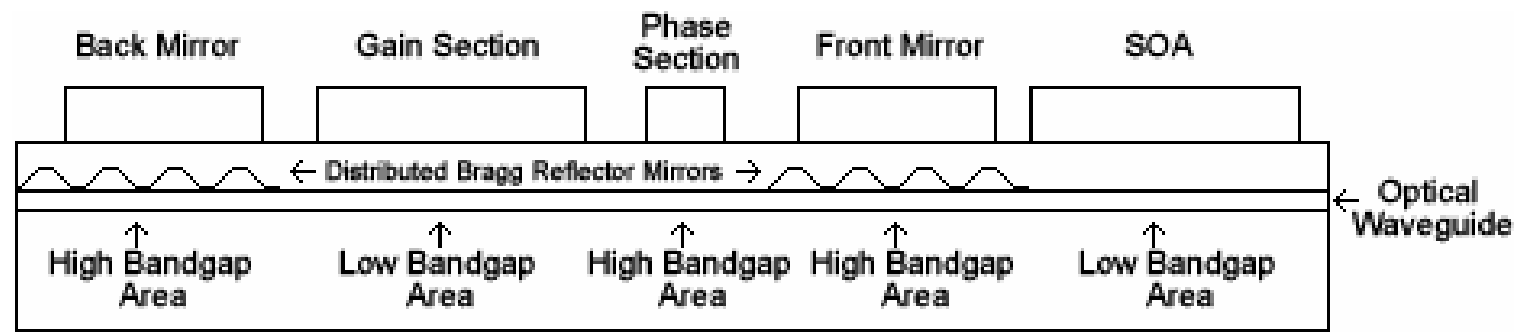

Figure 2: Cross sectional illustration of the SGDBR laser of figure 1. The gain section of the laser provides the required amplification for optical oscillation. The front mirror and back mirror provide wavelength dependent reflectivity to select the lasing wavelength of the laser. The phase section provides fine adjustments to the effective spacing between the mirrors. The semiconductor optical amplifier (SOA) provides boosts the output power from the laser. The back mirror, front mirror and phase sections utilize higher bandgap materials so that they are not absorbing at the lasing wavelength. Currents are applied to the laser segments to control the frequency and amplitude of the output.

The key concept for wavelength tuning this SGDBR laser lies with understanding the sampled Bragg grating that is placed below the Back Mirror and Front Mirror segments of the laser. Traditional Bragg reflector mirrors have a continuous grating whereas the SGDBR device has a periodic removal of the Bragg segment to form the more complicated reflectivity spectrum that is illustrated in Figure 3. 


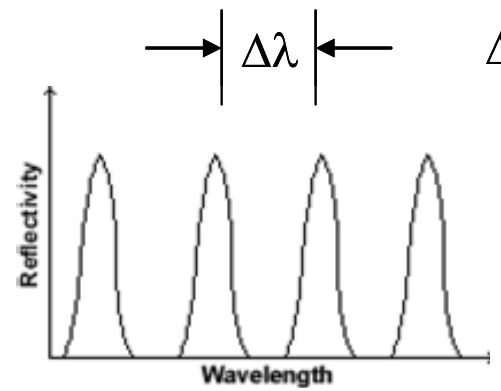

(a)

$\Delta \lambda=\lambda^{2} /(2 \mathrm{~nL})$

where $n=$ effective index of refraction of the waveguide

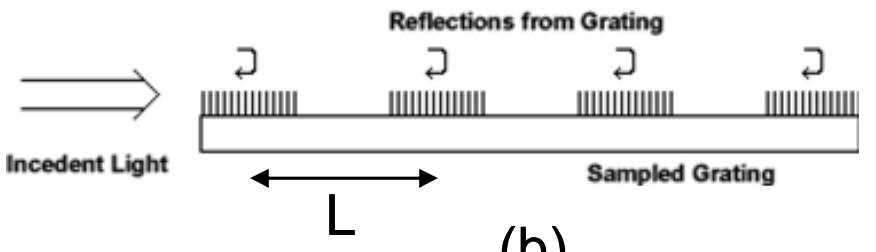

(b)

Figure 3: (a) The front mirror and back mirror reflectivity versus wavelength function is illustrated. The spacing between reflectivity peaks for this device is approximately $5 \mathrm{~nm}$. (b) The reflectivity versus wavelength function of part (a) is the result of a sampled Bragg grating structure. Here the Bragg gratings are constructed such that they have a broad reflectivity peak over the $1530 \mathrm{~nm}$ to $1560 \mathrm{~nm}$ range. The Bragg grating is not continuous though. The interrupted Bragg Grating structure introduces a Fabry-Perot interferometer between the individual Bragg grating reflectors resulting in the periodic reflectivity versus wavelength function. Given the $5 \mathrm{~nm}$ spacing between segments and an average waveguide index of refraction of 3.3, the spacing between the Bragg reflector segments is 72 microns. The periodic removal of the grating segments can be seen visually in figure 1 as vertical lines below the front mirror and back mirror metallization contacts.

The sampled Bragg Grating forms the frequency dependent reflectivity illustrated in figure 3a. The spacing, L, shown in figure $3 \mathrm{~b}$ is chosen to be slightly different between the front mirror and back mirror segments of the device. A vernier effect can then be used to tune the laser over a wide frequency range as is illustrated in figure 4.

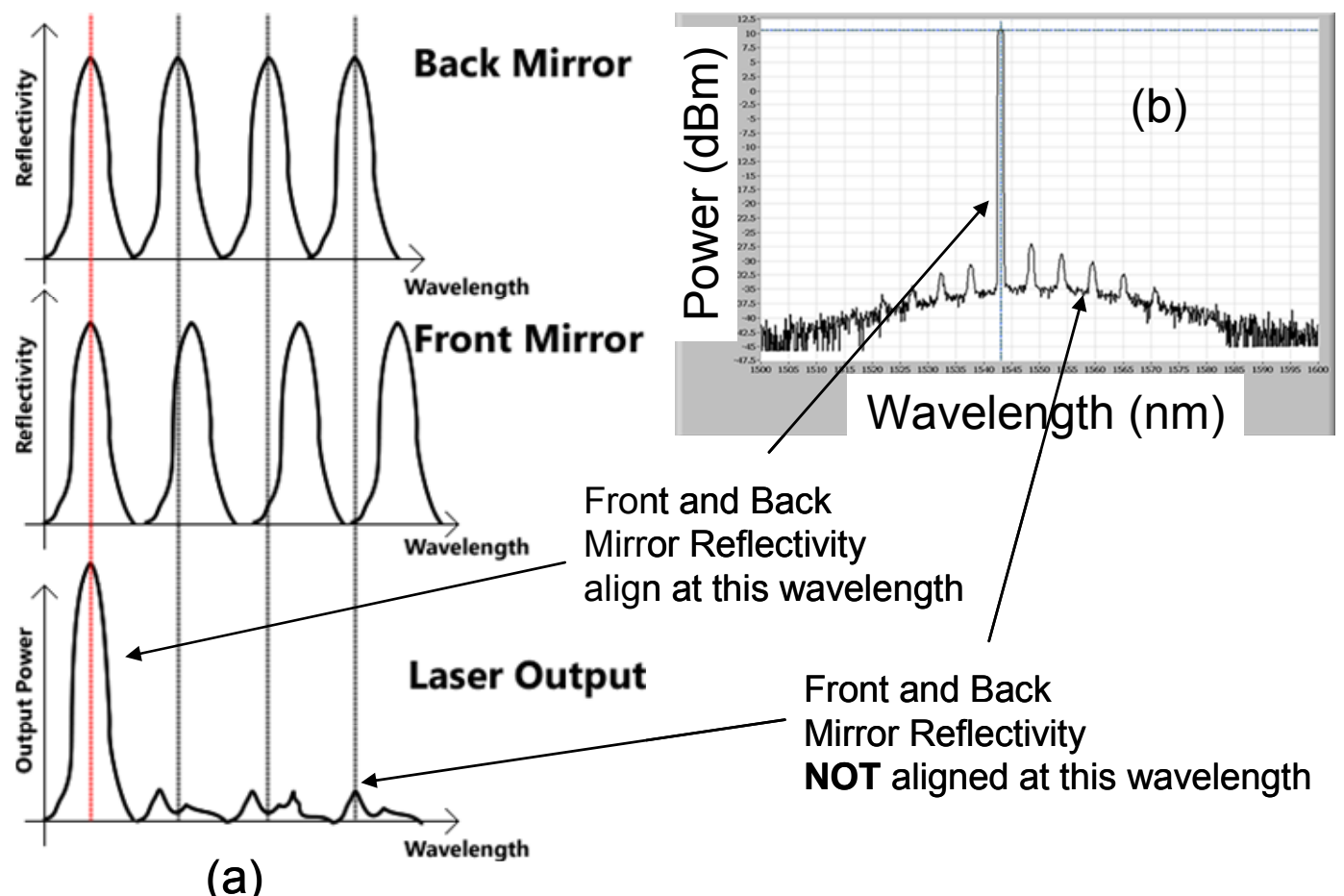

Figure 4: The wavelength of the SGDBR laser is controlled by alignment of the reflectivity peaks of the front and back mirrors. (a) The spacing between the interrupted gratings is slightly different in the front and back mirror resulting in a different periodicity in the reflectivity versus wavelength function. Only when the reflectivity peaks from the front and back mirror are aligned will the laser be able to oscillate. Part (b) of the figure shows a measured spectrum from an SGDBR laser. The laser shows one strong lasing mode and several small residual power peaks (typically $40 \mathrm{~dB}$ suppressed). The residual peak's periodicity corresponds to the reflectivity versus wavelength function of the front and back mirrors. The residual peaks are spaced at $5 \mathrm{~nm}$ in this device. 
Figure 4a shows the front mirror and back mirror with a slightly different periodicity in the reflectivity versus wavelength function. Only the first of the four reflectivity peaks is well aligned forcing the laser to oscillate at this wavelength. Current applied to the front or back mirror will change the carrier density in the segment. The carrier density in turn changes the index of refraction. Finally the index of refraction will move the position of the reflectivity peaks versus wavelength. For practical values of current excitation, each reflectivity peak can be moved by up to 5 $\mathrm{nm}$. Tuning over the entire amplification range of the gain section of the laser is accomplished by moving between the reflectivity peaks that are $5 \mathrm{~nm}$ spaced from each other. Figure 5 shows an example tuning curve as one varies the current in the back mirror and the front mirror of the laser.

\section{Tuning Characteristics: Agility 1107}

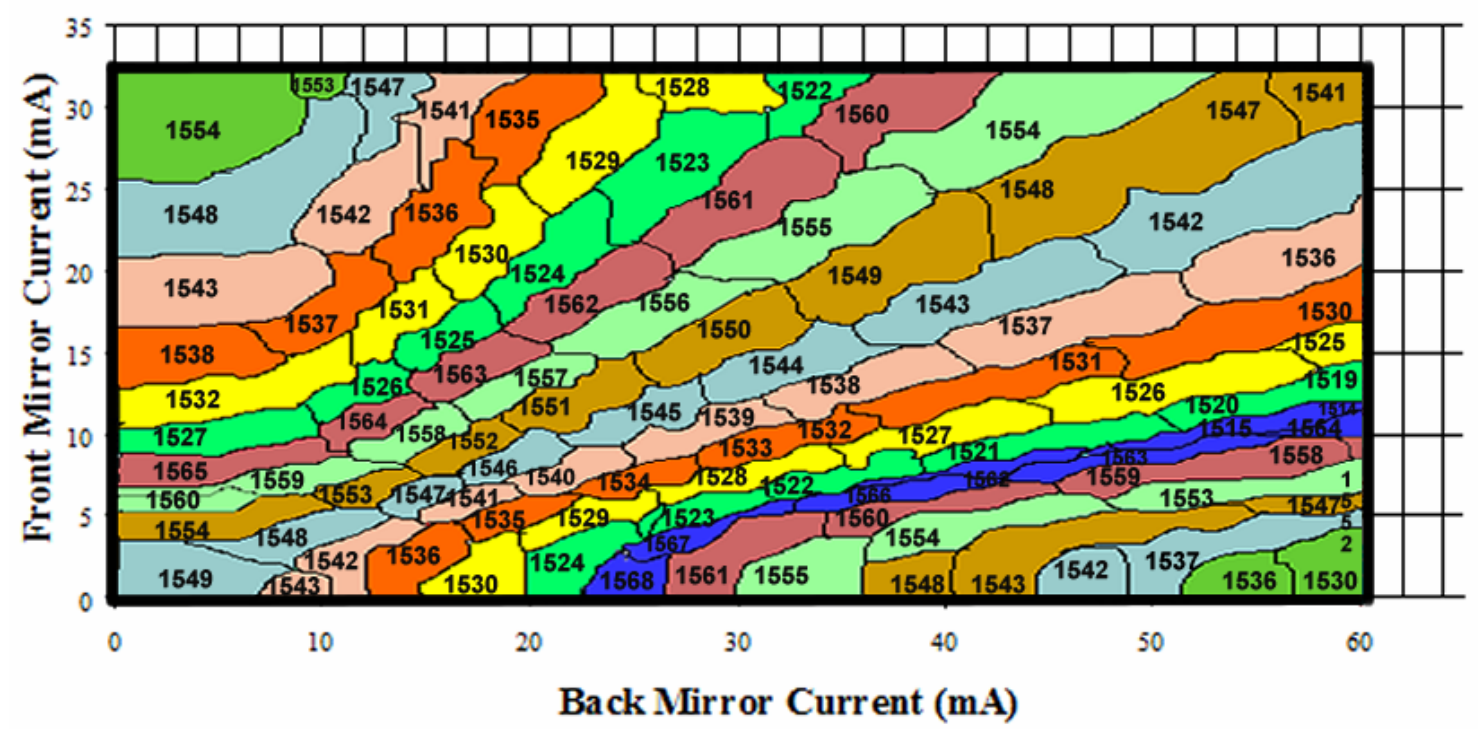

Figure 5 - An illustration of the wavelength tuning characteristics of the SGDBR laser. The wavelengths where the laser is operating on the same set of front mirror and back mirror reflectivity peaks are shown in a common color shade. Each wavelengthlabeled shape segment represents an area where the lasing wavelengths are within a $1 \mathrm{~nm}$ of each other.

Figure 5 graphically illustrates the boundaries of each of the $5 \mathrm{~nm}$-spaced reflectivity peaks shown in figure 4 . The laser oscillates at $1549 \mathrm{~nm}$ with no current applied to either the front or back mirror. A continuous change in wavelength can be achieved by simultaneously increasing the current in each mirror and following a diagonal path from the $1549 \mathrm{~nm}$ segment up to $1542 \mathrm{~nm}$ shape in the upper right hand corner of the diagram. This path represents a continuous wavelength sweep path where both the front mirror and back mirror reflectivity peaks slide along in wavelength together. Note that the front and back mirror segments are higher bandgap material segments (see figure 2) so that they are low loss even without applied current. The phase section of the laser can also control the wavelength of the laser. The current applied to the phase section controls the delay through the phase section allowing for a control of the cavity laser length and thus the oscillation wavelength. The phase section essentially allows for fine tuning control on the laser frequency with a typically sensitivity of $1 \mathrm{GHz} / \mathrm{mA}$ of tuning current change. For OCT measurements, it is necessary to tune over the entire frequency range of the laser in order to get maximum distance resolution in the measurement. Figure 6 shows how the entire tuning range of the laser can be obtained by following adjacent diagonal paths through the tuning curve of figure 5. Figure 6 shows how the laser first continuously tunes across a reflectivity peak and then jumps to an adjacent reflectivity peak at the same wavelength to continue the wavelength ramp. The caption of the figure highlights a series of paths that could be followed in a concatenated wavelength ramp. Figure 7 shows the current versus time waveform that is needed in the front and back mirror segments to got through a series of 5 laser modes in covering the 1548 to $1568 \mathrm{~nm}$ wavelength range. Although the entire wavelength range is tuned with this method, the tuning algorithm is quite complex. At least two coordinated non-linear ramping currents are needed in order to construct a linear tuning ramp versus time. Although this is a design hurdle, there are arbitrary waveform generation circuits that can provide these current versus time functions in a fast wavelength sweep. 


\section{Tuning Characteristics: Agility 1107}

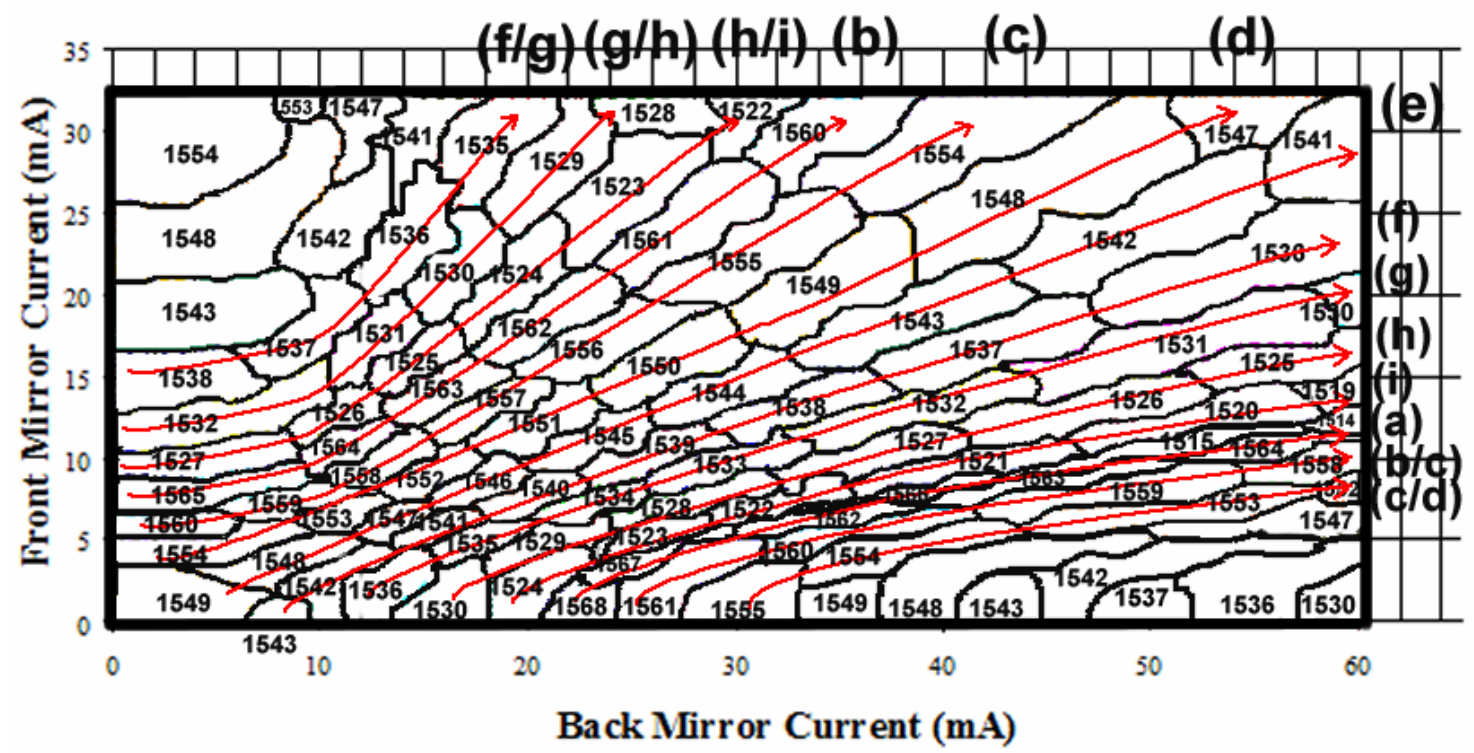

Figure 6 - The tuning algorithm for an SGDBR wavelength ramp is outlined. An example path for current control necessary to produce a tuning ramp is illustrated with the following path sequence: (a) Starting path $1568 \mathrm{~nm}-1564 \mathrm{~nm}$. (b) $1564 \mathrm{~nm}-1560 \mathrm{~nm}$. (c) $1559 \mathrm{~nm}-1554 \mathrm{~nm}$. (d) $1552 \mathrm{~nm}-1547 \mathrm{~nm}$. (e) $1546 \mathrm{~nm}-1541 \mathrm{~nm}$. (f) $1540 \mathrm{~nm}-1536 \mathrm{~nm}$. (g) $1535 \mathrm{~nm}-1530 \mathrm{~nm}$. (h) $1529 \mathrm{~nm}-$ $1525 \mathrm{~nm}$. (i) $1524 \mathrm{~nm}-1520 \mathrm{~nm}$.

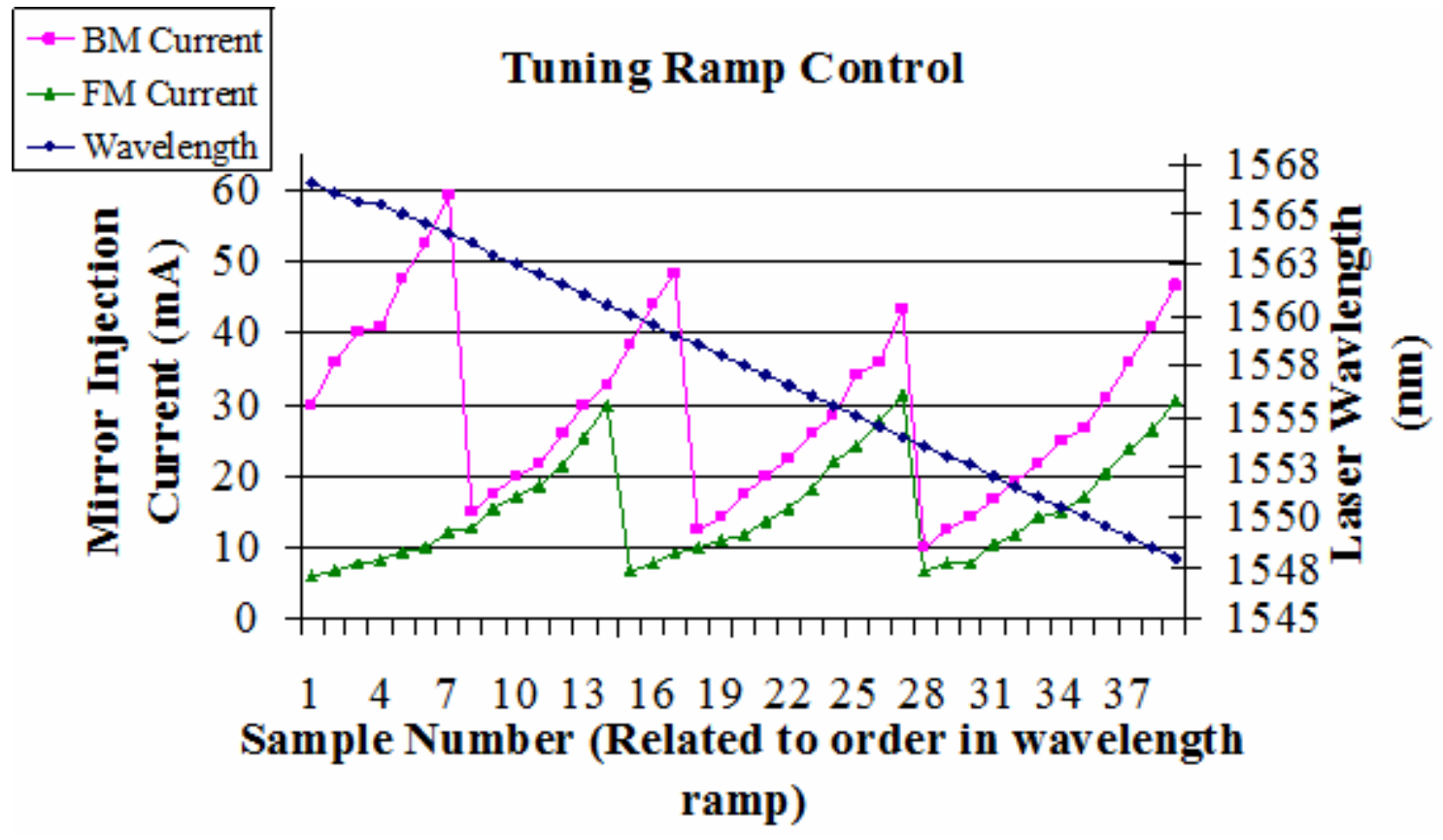

Figure 7 - Example currents required to produce a linear wavelength ramp between $1548 \mathrm{~nm}$ and $1568 \mathrm{~nm}$ are given. The front mirror (FM) and back mirror (BM) currents are non linear versus time and will need to be generated with an arbitrary waveform generator.

This investigation on SGDBR lasers for OCT applications has focused on open-loop tuning of the laser wavelength. Open loop means that feedback generated by comparison to an optical frequency reference is not used when ramping the laser wavelength. Open loop tuning requires repeatability of the laser wavelength ramp when the drive currents are applied repeatedly. An investigation on the repeatability of the tuning ramp was done. It was found that wavelength 
repeatability issues were observed in wavelength tuning regions that were on the boundaries between the $5 \mathrm{~nm}$ wavelength jumps found in figures 5 and 6 . These $5 \mathrm{~nm}$ wavelength jump boundaries were found to be exhibit hysteresis as illustrated in figure 8 .
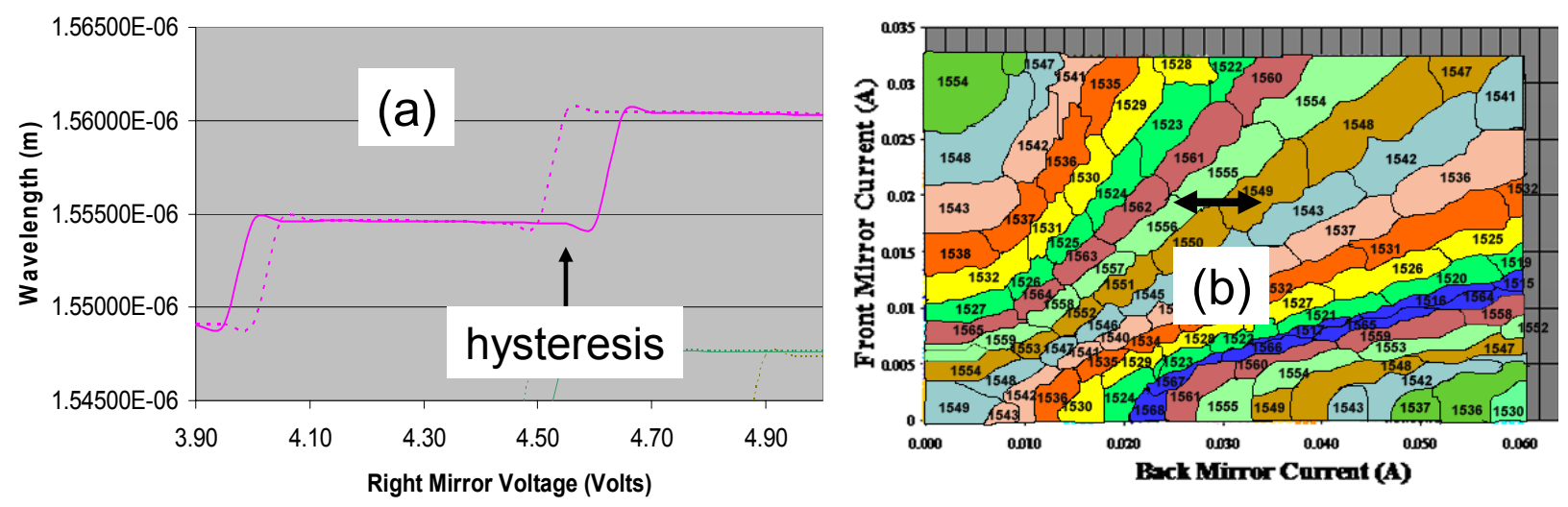

Figure 8: (a) Hysteresis occurs when reflectivity peak boundaries are crossed. Here the current to the mirror was increased in the solid line when crossing a boundary between reflectivity peaks. The dashed line shows the wavelength as the current is decreased across the same reflectivity peak boundary. (b) The arrow on this curve indicates the boundary that was crossed in figure 6 a.

The crossing of the $5 \mathrm{~nm}$ switching boundary illustrated by the arrow of figure $8 \mathrm{~b}$ is a function of the direction of crossing. Figure 8a show the wavelength as a function of current as the current is increased or decreased when crossing the boundary. The effects of hysteresis are clearly visible If reliable open-loop tuning is desired, these modehop regions need to be avoided. There are additional effects that must be accounted for when the wavelength is quickly swept over wavelength including temperature transients in the laser and electrical circuit charging effects.

\section{Tuning Ramp (64 Points)}

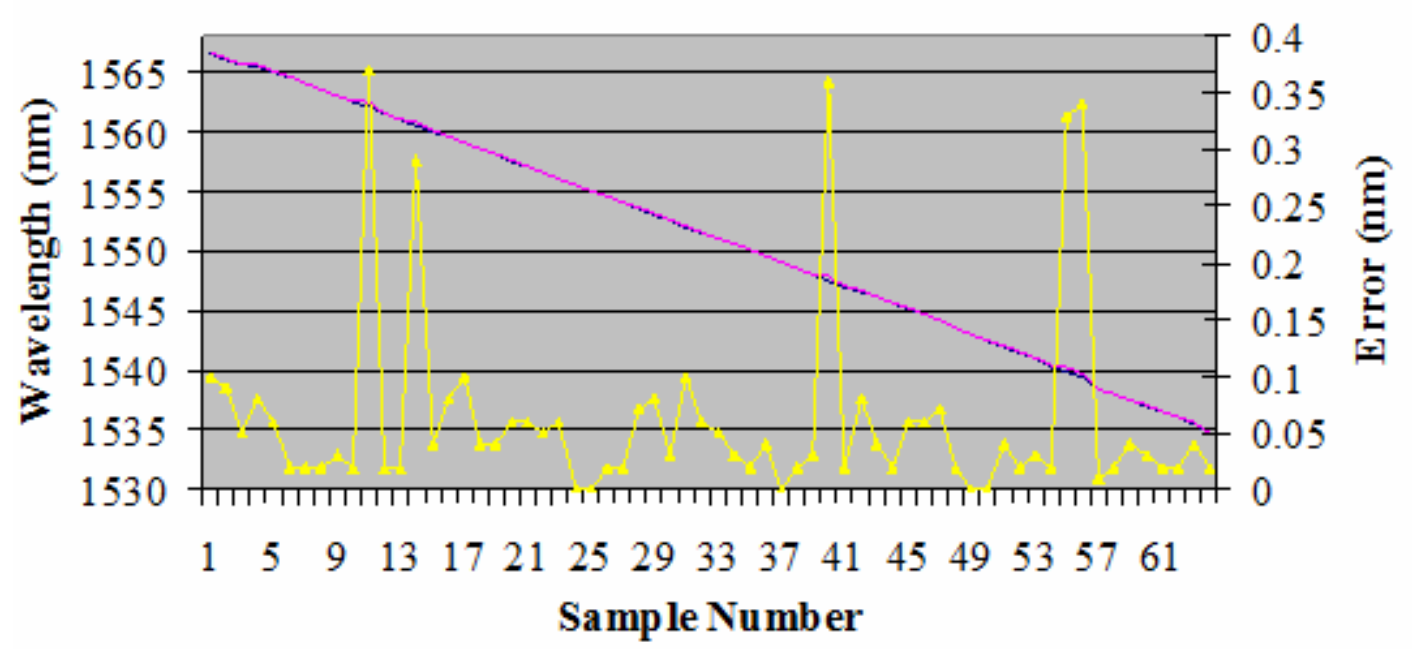

Figure 9 - Reproducible tuning ramp results are shown. The desired wavelength ramp was repeated over a two week time interval. The two wavelength versus sample number curves are very close to each other. The difference between these two measurements is also shown.

Figure 9 shows the results of an open-loop repeatability test that was done over a 2 week time interval. Most of the wavelengths repeated within $0.05 \mathrm{~nm}$ with a few deviating by as far as $0.35 \mathrm{~nm}$. All of the $5 \mathrm{~nm}$ mode jumps caused by hysteresis have been eliminated by using the tuning paths illustrated in figures 6 and 7 . Investigation still continues 
on improving the results of figure 9 by a re-design of the electronic drive hardware and software. All of the results illustrated so far are for slow tuning ramps. The ultimate goal of this investigation is to be able to take advantage of the fast wavelength switching capability of the laser for OCT applications. These lasers were originally designed for telecommunications application where it was not necessary to switch the laser wavelength quickly. Most telecommunication applications utilize integrated low pass filters into the laser package in order to minimize the potential for wavelength drift after the wavelength of the laser has been set to a communication channel location. In order to explore fast wavelength switching two approaches were taken as illustrated in figure $10 \mathrm{a}$ and $\mathrm{b}$. First individual SGDBR laser chips were mounted an AlN Carrier and then wirebonded to $50 \mathrm{ohm}$ microstrip lines fabricated on an Alumina substrate. Customized butterfly laser packages were also utilized that had internal low-pass filters removed to allow fast wavelength switching.

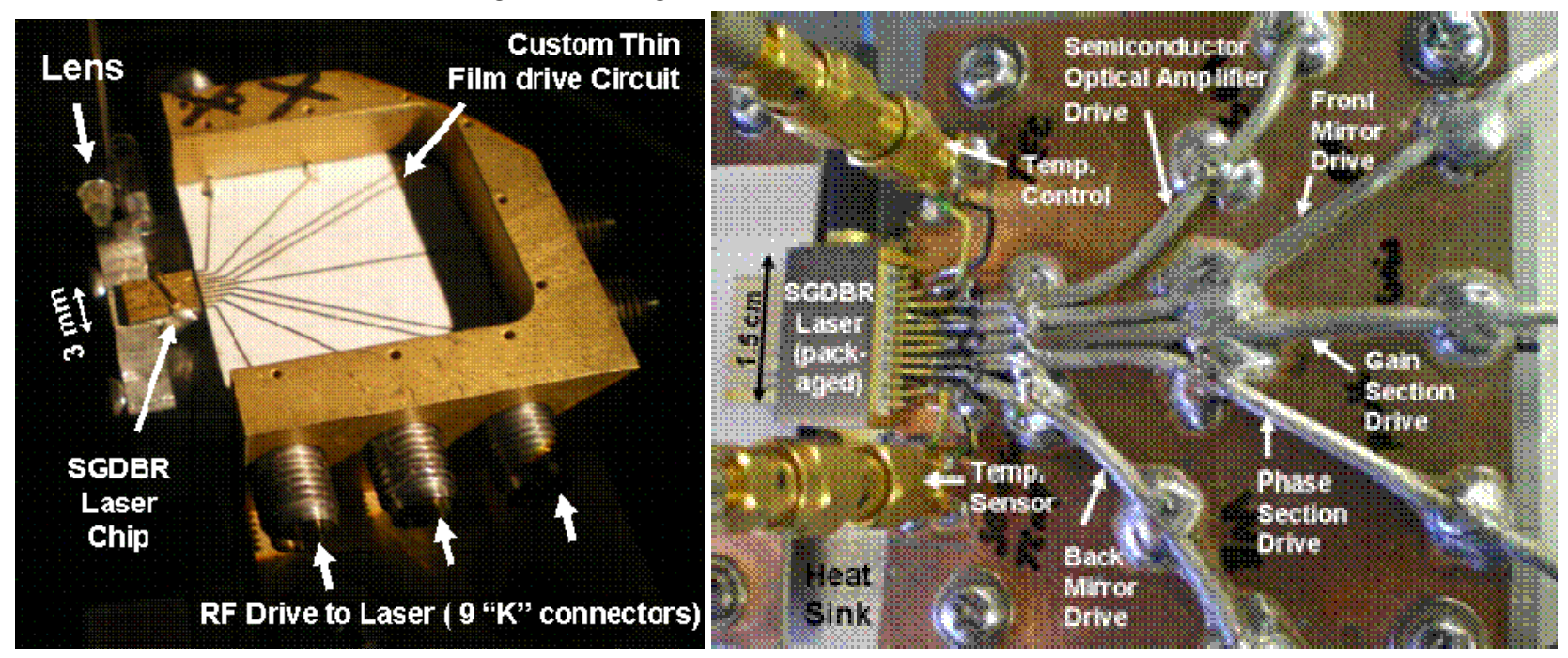

Figure 10. (left) This is a picture of a SGDBR laser chip connected to a series of $50 \mathrm{ohm}$ microstrip lines an alumina substrate leading to high speed K-connectors to allow high speed modulation of frequency and amplitude. (right) This picture shows a butterfly packaged chip with internal low pass filters removed. The leads are fed with 0.085 inch diameter semirigid coaxial cable to allow high speed drive of the packaged device.

Figure 10b shows semi-rigid coaxial cables that were connected to the pins of the butterfly package to reduce the parasitic electrical effects of the package leads. The butterfly leads were also suspended over a dielectric to produce a structure that behaved closer to a $50 \mathrm{ohm}$ transmission line connection.

Previous research into SG-DBR lasers has led to demonstrations of fast wavelength switching capabilities of fewer than 5-ns for a 64-channel laser with switching accuracy of $\pm 12 \mathrm{GHz}$ [2]. The frequency modulation response of the laser was tested to determine the wavelength switching speed that was possible with this packaged devic of figure $10 \mathrm{~b}$. Figure 11 shows an example measurement of the packaged SGDBR laser device illustrated in figure 10b. A sinusoidal current was applied to the phase section of the laser inducing sinusoidal frequency modulation on the laser. The packaged SGDBR laser output was applied to a tunable optical filter. The optical filter center frequency was adjusted so that the laser was located offset from the wavelength of maximum transmission of the SGDBR signal. The optical filter was utilized as a frequency discriminator filter to convert frequency modulation into amplitude modulation. This FM modulation frequency response curve shows that the package can be effectively modulated up to $100 \mathrm{MHz}$. Using a time-bandwidth product of 1 , this would imply that wavelength switching times under 10nS should be possible with this packaged device.

The linewidth of the SGDBR laser is one of the concerns for its applicability to OCT applications. Figure 12 shows an example measurement of the laser linewidth using a delayed homodyne technique ${ }^{5}$. This measurement is taken for the laser with no modulation applied to the laser. Since the front mirror, back mirror and phase section of the laser affect the wavelength, the segments are vulnerable to any electric noise current that appears on these terminals. 


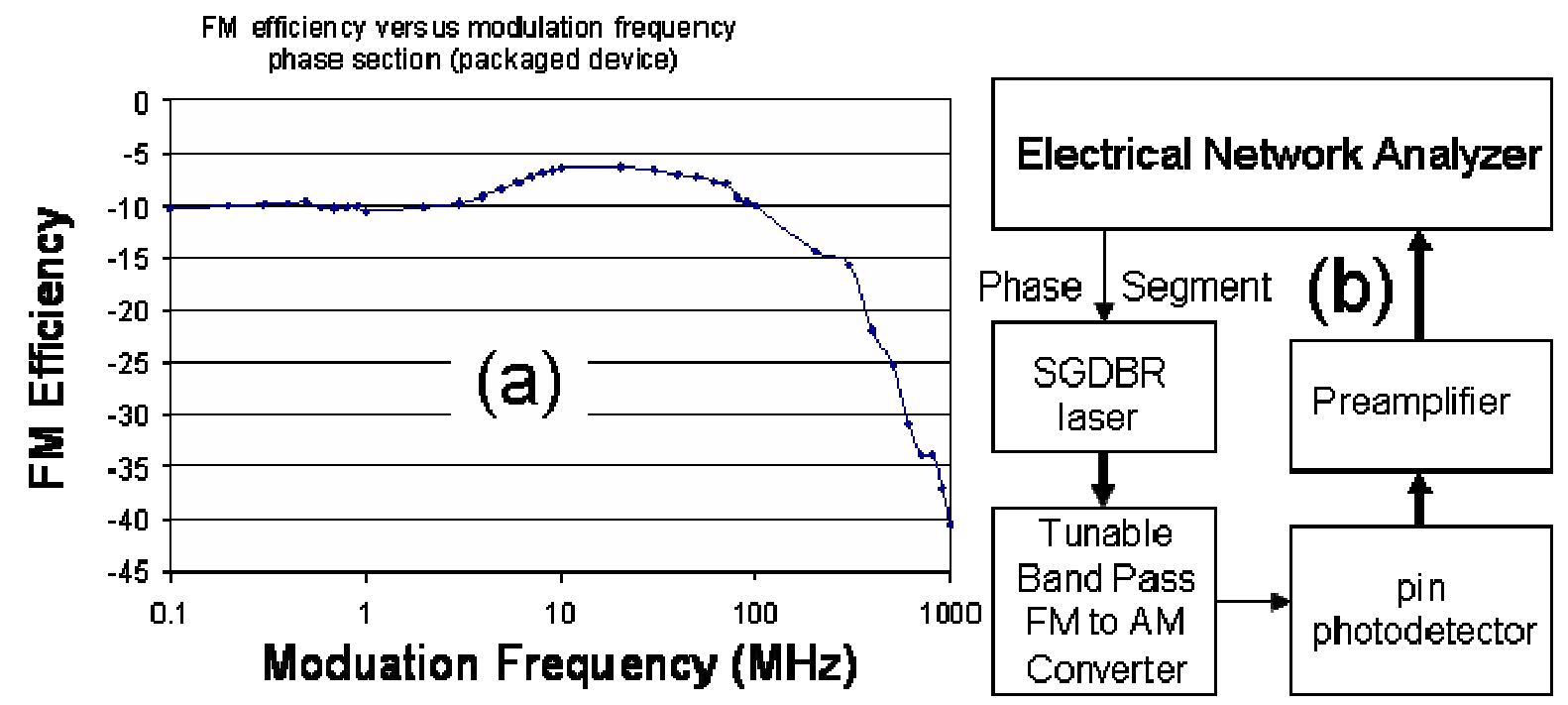

Figure 11: (a) The FM efficiency of the Phase Segment of the SGDBR laser is shown as a function of modulation frequency. The $-3 \mathrm{~dB}$ electrical bandwidth of the FM modulation for the packaged devic of figure 10b was $100 \mathrm{MHz}$.. (b). The FM modulation efficiency measurement block diagram is shown. An AC current was applied the phase section of the packaged laser. The frequency modulated output of the laser is then applied to the skirt of a narrow bandpass filter to form an FM to AM converter. The resulting amplitude modulation is then detected in a pin photodetector. Finally the amplified signal is compared to the input electrical modulation in the network analyzer.
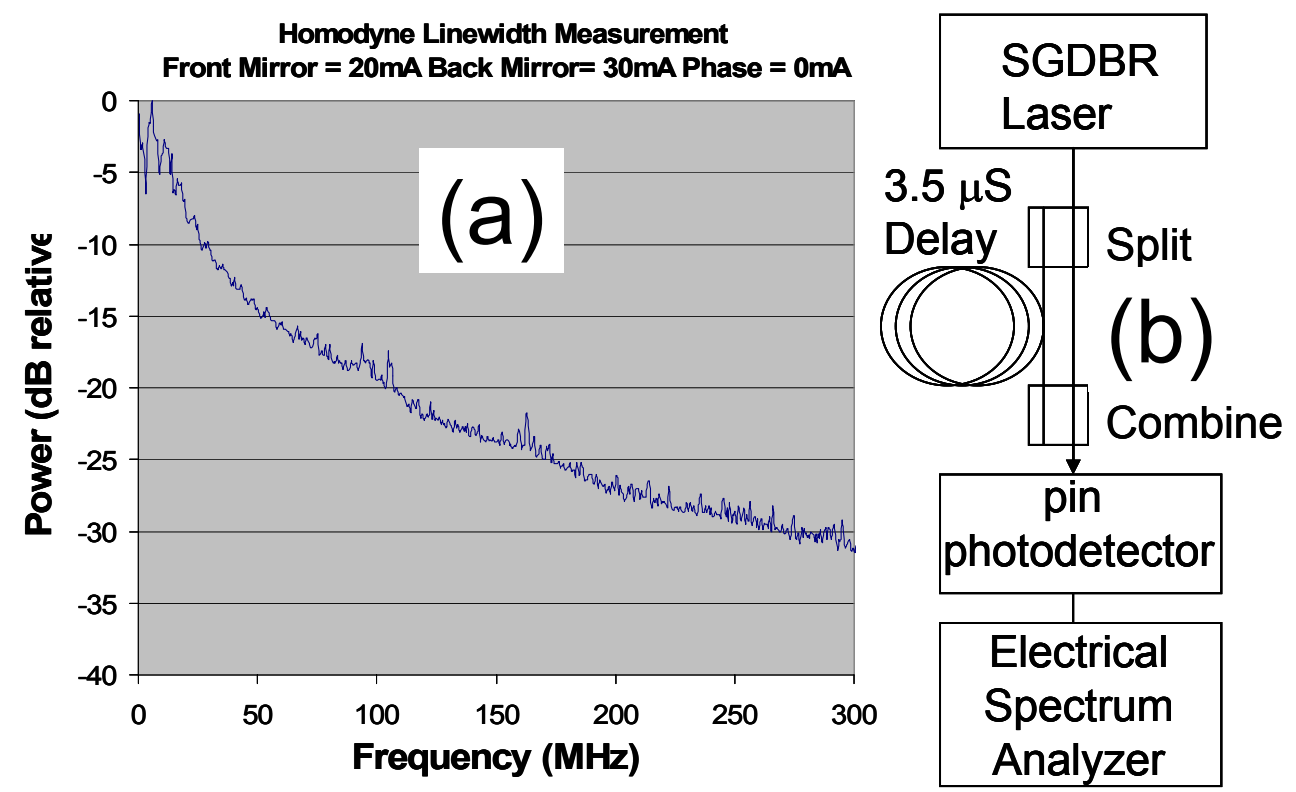

Figure 12: (a) A homodyne linewidth measurement of the SGDBR laser is shown. The laser is unmodulated. (b) The experimental apparatus for measuring the linewidth by homodyne techniques.

Work continues on increasing the ramp rate and stitching together the tuning curve segments illustrated in figure 6 using fast electrical current driving waveforms consisting of phase-locked arbitrary waveform generators with open 
loop wavelength control. Temperature transients in the laser and charging time constants in the device are being compensated for by adjustments in the current drive waveforms to the laser.

The next section of this paper talks about an early system experiment that was done in order to investigate architectures that utilize the SGDBR laser for OCT applications. The purpose of this first experiment is as a building block to future OCT system architectures that are optimized for the SGDBR capabilities.

\section{SYSTEM EXPERIMENT}

There are many architectures that can be used to accomplish swept wavelength OCT. The FM-CW technique is commonly used in many experiments ${ }^{5}$. Variations on this architecture are being evaluated for how well they fit with the SGDBR strength and weaknesses as highlighted in table 1 and table 2.

As a simple starting point, we did a system-level evaluation of the SGDBR laser using an architecture that is commonly used to measure chromatic dispersion (wavelength dependent time delay in optical fiber). The architecture of the measurement is shown in figure 12. This modulation phase shift technique ${ }^{5}$ utilizes an optical modulator to amplitude modulate the output of the SGDBR laser. Versions of the SGDBR laser exist where the amplitude modulation function is accomplished on the same laser chip. An electrical network analyzer is used to drive the amplitude modulator. The modulated optical signal is then applied to a device under test which in this case is simply a length of optical fiber. The output of the device under test is then photodetected and applied to the network analyzer through an electronic preamplifier. The phase and magnitude of the electrical drive to the network analyzer is compared to the received modulation at the output of the device under test. The magnitude and phase are compared as a function of frequency in the electrical vector network analyzer. Group delay changes as a function of optical frequency in the device under test are calculated by taking the derivative of the phase (in radians) with respect to the change in optical frequency (radians/second).

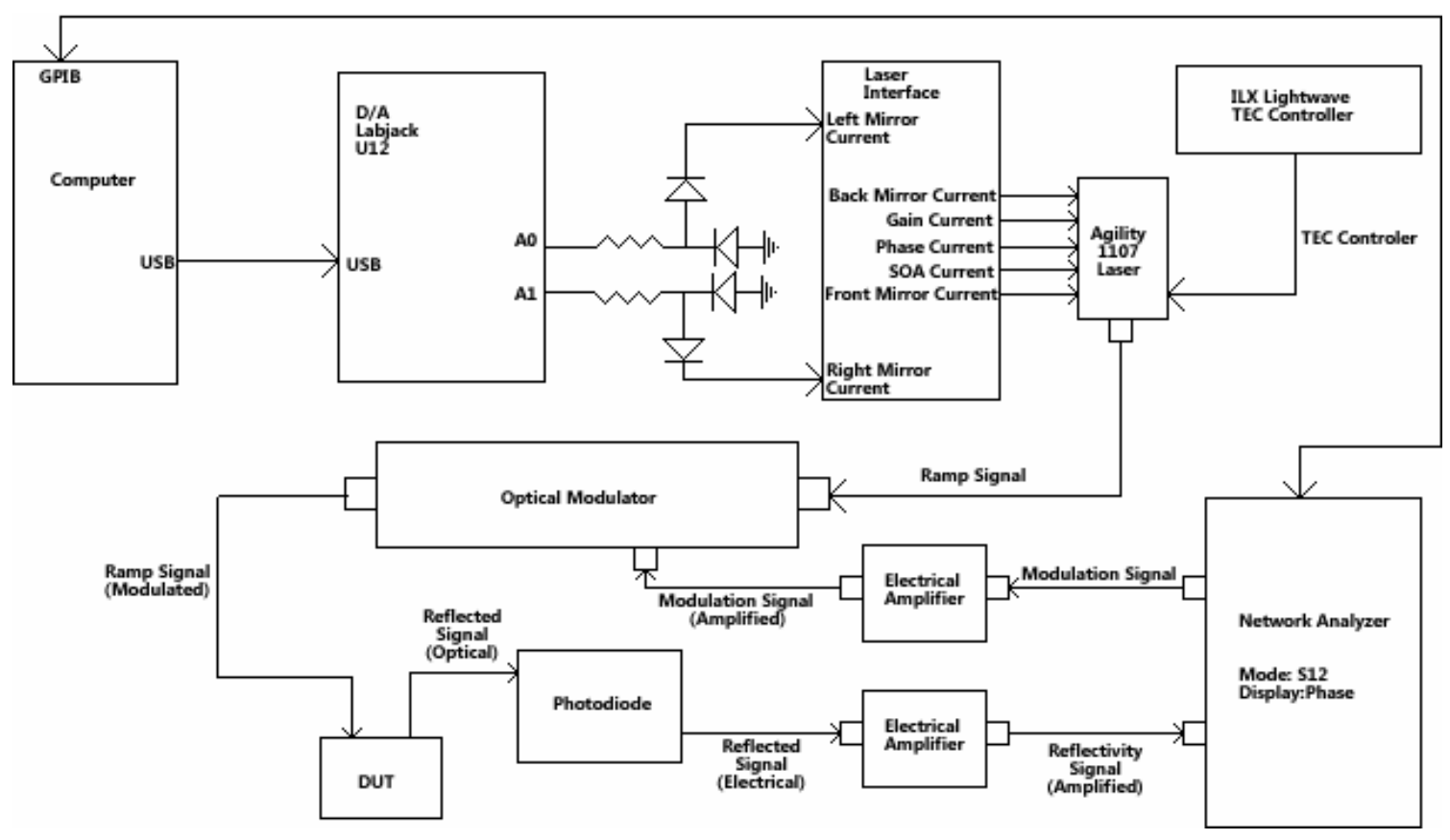

Figure 12: (a) The modulation phase shift technique of measuring delay versus wavelength is illustrated. This was a first experiment used to evaluate the SGDBR laser at the system level for OCT applications. 


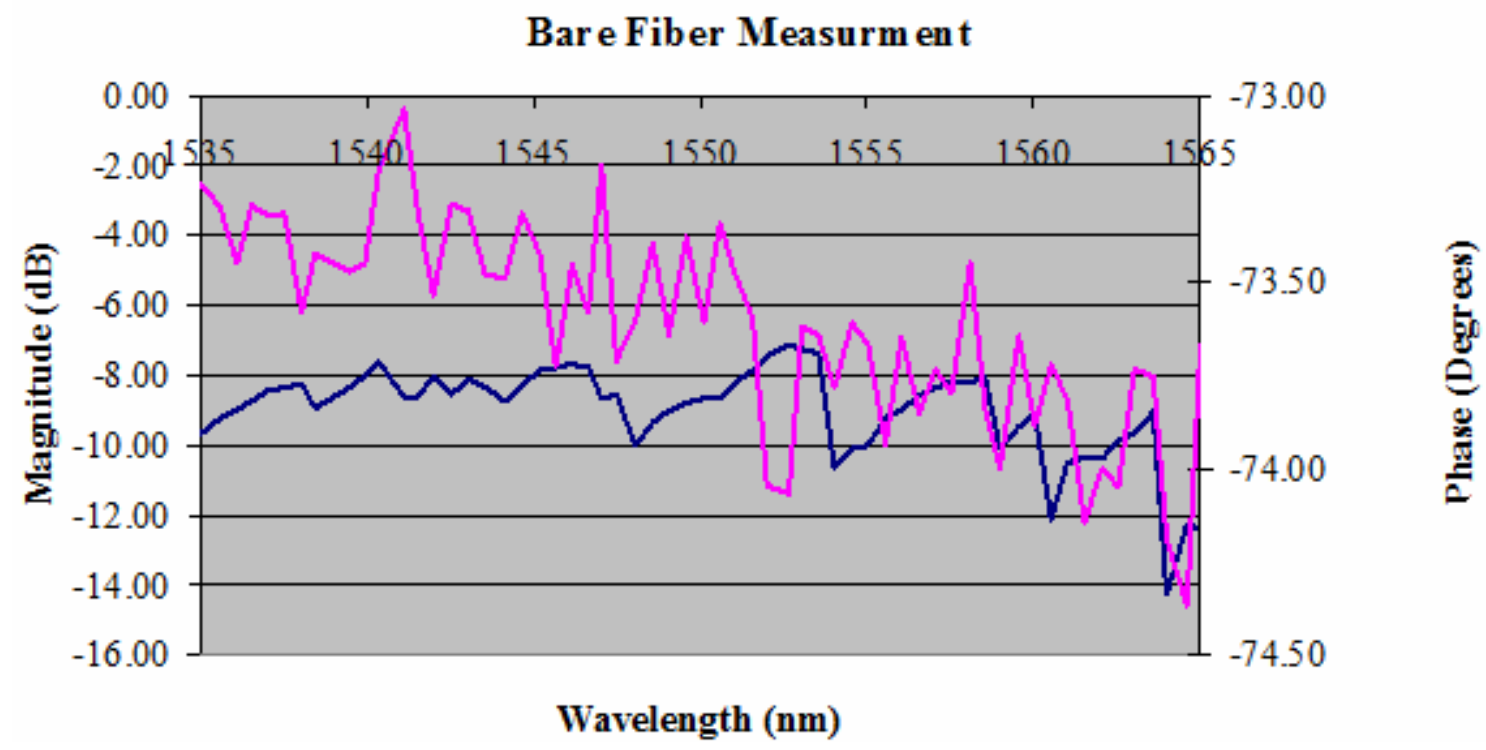

Figure 13 - An example measurement of a short length of $9 / 125 \mu \mathrm{m}$ single mode telecommunications fiber as a device under test.

Figure 13 shows and example measurement results for short length of optical fiber. Since the fiber lengths was very short, the change if phase with respect to frequency (or group delay) is relatively constant with respect to wavelength.

\section{SUMMARY AND FUTURE WORK}

The strengths and the weakness of the SGDBR laser for swept wavelength OCT measurements were highlighted. A summary of SGDBR laser structure was then given to illustrate the mechanism for wavelength tuning. Three segments of the laser, the Front Mirror, Back Mirror and Phase sections of the laser can change the laser wavelength as a function of applied current. The low repetition rate wavelength tuning map of the laser was then demonstrated. A current tuning algorithm was proposed and evaluated to allow the laser to ramp over the devices tuning range. This tuning ramp requires several current control segments to be simultaneously varied. Two laser packaging styles were constructed to allow high frequency wavelength tuning of the lasers. Swept sinusoidal measurements of the FM response show a $100 \mathrm{MHz}-3 \mathrm{~dB}$ frequency response demonstrating the fast wavelength switching response of the packaged laser. Finally a first system level experiment was done to understand some of the higher level architectural issues of using this laser in a swept-wavelength OCT application.

The next phase of this work involves using phase-locked arbitrary waveform generators to produce a high speed wavelength ramp. Issues associated with open-loop wavelength control and wavelength repeatability will be addressed. Laser heating effects and electrical parasitic charging effects will add additional complexity to the current drive waveforms. The goal will be to produce a system where the user is abstracted away from the detailed mechanisms of tuning the wavelength ramp. Variations on the FM-CW OCT measurement technique will then be performed using to evaluate system level performance when optimized to the SGDBR laser capabilities. The limitations of the sources spectral linewidth will also be evaluated.

\section{ACKNOWLEDGEMENTS}

The authors would like to acknowledge funding from the central coast research program at Cal Poly for funding this work along with device donations from JDSU. Matt DiGino and Sam McGinn were also early senior-project contributors to this work. 


\section{REFERENCES}

[1] L.A. Johansson, Y.A. Akulova, G.A. Fish, and L.A. Coldren, "Sampled-Grating DBR Laser Integrated with SOA and Tandem Electroabsorption Modulator for Chirp-Control," Electronics Letters, 40, pp. 70-71 (January 2004)

[2] John E. Simsarian, Michael C. Larson, Henry E. Garret, Hon Xu, and Timothy Strand " Less than 5-ns Switching with an SG-DBR Laser", IEEE Photonics Technology Letters, Volume 18, No. 4, pages 565-567 (February 2006).

[3] J. Buus, E. J. Murphy, Tunable Lasers in Optical Networks, vol. 24, Journal of Lightwave Technology, 2006, p. 5

[4] L. A. Coldren, G. A. Fish, Y. Akulova, J. S. Barton, L. Johansson, C. W. Coldren, Tunable Semiconductor Lasers: A Tutorial, vol. 22, Journal of Lightwave Technology, 2004, p. 193

[5] Dennis Derickson (Editor), Fiber Optic Test and Measurement, ISBN 0-13-534330-5, Prentice Hall, 1998. See chapter 10 (pages 425-429) for a discussion on FMCW chirped-laser techniques. 